\title{
Parallelism of DOG1 expression with recurrence risk in gastrointestinal stromal tumors bearing KIT or PDGFRA mutations
}

Francesca Maria Rizzo ${ }^{1 *}$, Raffaele Palmirotta ${ }^{1,2}$, Andrea Marzullo ${ }^{3}$, Nicoletta Resta ${ }^{4}$, Mauro Cives $^{1}$, Marco Tucci ${ }^{1}$ and Franco Silvestris ${ }^{1}$

\begin{abstract}
Background: Gastrointestinal stromal tumors (GISTs) are characterized by mutations of KIT (v-kit Hardy-Zuckerman 4 feline sarcoma viral oncogene homolog) or PDGFRA (platelet-derived growth factor receptor a) that may be efficiently targeted by tyrosine kinase inhibitors (TKI). Notwithstanding the early responsiveness to TKI, the majority of GISTs progress, imposing the need for alternative therapeutic strategies. DOG1 (discovered on GIST-1) shows a higher sensitivity as a diagnostic marker than KIT, however its prognostic role has been little investigated.
\end{abstract}

Methods: We evaluated DOG1 expression by immunohistochemistry (IHC) in 59 patients with GISTs, and correlated its levels with clinical and pathological features as well as mutational status. Kaplan-Meier analysis was also applied to assess correlations of the staining score with patient recurrence-free survival (RFS).

Results: DOG1 was expressed in $66 \%$ of $\mathrm{CD} 117^{+}$GISTs and highly associated with tumor size and the rate of wildtype tumors. Kaplan-Meier survival analysis showed that a strong DOG1 expression demonstrated by $\mathrm{IHC}$ correlated with a worse 2-year RFS rate, suggesting its potential ability to predict GISTs with poor prognosis.

Conclusions: These findings suggest a prognostic role for DOG1, as well as its potential for inclusion in the criteria for risk stratification.

Keywords: Gastrointestinal stromal tumors, DOG1, Size, Mutation, Prognostic value, Risk

\section{Background}

Gastrointestinal stromal tumors (GISTs) develop within the digestive tract and harbor functional mutations of KIT (v-kit Hardy-Zuckerman 4 feline sarcoma viral oncogene homolog) and PDGFRA (platelet-derived growth factor receptor- $\alpha$ ) that primarily drive the tumor growth and progression [1, 2]. KIT and PDGFRA genes are located on the chromosome 4q12 and encode transmembrane glycoproteins belonging to the type III receptor tyrosine kinase family. They are normally activated by their ligands, namely stem cell factor and PDGF respectively, which bind the receptor extracellular domain leading to the dimerization of receptors and phosphorylation of tyrosines in their cytoplasmic tyrosine

\footnotetext{
* Correspondence: frarizzo3@libero.it

'Department of Biomedical Sciences and Human Oncology, University of Bari

"A. Moro", Piazza Giulio Cesare, 11-70124 Bari, Italy

Full list of author information is available at the end of the article
}

kinase (TK) domains in a process called signal transduction. This triggers a phosphorylation cascade of the tyrosine residues in multiple downstream molecules and leads to the activation of signal transduction pathways involved in many important cell functions such as proliferation, apoptosis, chemotaxis and adhesion [3]. The presence of KIT and PDGFRA activating mutations provides the rationale for employing targeted therapies using specific inhibitors (TKI), that can improve recurrence-free survival (RFS) and overall survival (OS) in the majority of patients. The currently used systems for risk stratification are based on tumor size and site, mitotic count and tumor rupture, whereas the prognostic relevance of mutational status is still under debate [4]. CD117 expression occurs in more than $95 \%$ of GISTs bearing KIT or PDGFRA mutations [5], the remaining $5 \%$ are either CD117 negative or wild-type (WT) for both genes. Thus, to obtain a definite diagnosis additional morphological and/or molecular 
characterization may be required, such as searching for germline or de novo mutations of $S D H$ (succinate dehydrogenase) subunits located on the inner membrane of the mitochondria, or even mutations of the RAS-pathway [6]. Among the latter, the frequency of $B R A F$ mutations varies from 2 to $13 \%$, whereas $K R A S$ mutations are extremely rare $(<0.2 \%)$. Interestingly, concomitant $K R A S$ mutations in KIT- or PDGFRA-mutated GISTs were reported and, based on in vitro experiments, it has been defined that the presence of $R A S$ mutations predicts resistance of KIT-mutated GISTs to TKI [7]. However, two subsequent analysis in large cohort of GIST patients have not found mutations in KRAS codons 12 and 13 or $61[8,9]$. More recently, one single KIT/PDGFRA WT GIST was identified to carry a KRAS mutation in codon 12 among 267 patients and associated with an aggressive behavior and resistance to multiple TKI inhibitors [10].

DOG1 (Discovered on GIST-1) is a calcium-dependent chloride channel protein regulating the cholinergic activity of gastrointestinal smooth muscle [11] that is encoded by ANO1/TMEM16A on chromosome 11q13; in these tumors its expression shows high sensitivity and specificity $[12,13]$. Other functions exerted by ANO1 include the regulation of both the viability and proliferation of cells overcoming their checkpoints within the cell-cycle [14]. In addition, in DOG1 ${ }^{+}$cells ANO1 activates alternative signals downstream of the RAS/RAF/ MEK/ERK and the insulin-like growth factor (IGF)dependent pathways $[15,16]$. These findings support the hypothesis that DOG1 exerts a definite role in GIST development, regardless of KIT and PDGFRA activation, whereas its prognostic role is still debated.

Particularly in GISTs lacking CD117 expression and bearing PDGFRA mutations [17, 18], DOG1 appears to be a promising tool for diagnosis also of rare variants including gastric spindle and epithelioid-cell PDGFRAmutated GISTs [19]. However, its expression has been little correlated with other risk factors [20-22].

Here we explored the prognostic role of DOG1 in a cohort of patients with GISTs, and evaluated the potential correlation between variable grades of expression and known risk factors for recurrence.

\section{Methods}

\section{Patients and specimens}

Demographic data, histological and immunohistochemical features, as well as mutational status, of 59 patients with GISTs, enrolled at the Medical Oncology Unit of the University of Bari and the IRCCS San Raffaele Pisana in Rome from 2007 to 2014, were collected after obtaining patients' written informed consent and approval by the Ethics Committee of the University of Bari and the Ethics Committee of IRCCS San Raffaele Pisana in Rome, in accordance with the principles embodied in the Declaration of Helsinki. Selected hematoxylin/eosin stained slides were reviewed to confirm the diagnosis, as well as tumor features including size and histology; CD117 expression was evaluated by immunohistochemistry (IHC). In each sample the number of mitoses was evaluated in 50 consecutive high-power fields (HPFs), while demographic data including tumor staging at diagnosis and follow-up were retrieved from medical records.

\section{Mutational analysis of PDGFRA or KIT genes}

Tumor specimens were screened for hot-spot mutation sites of PDGFRA (exons 12 and 18) and KIT (exons 9, 11, 13 and 17) genes. To this end, genomic DNA was isolated from formalin-fixed, paraffin-embedded (FFPE) tissues containing at least $70 \%$ of neoplastic cells. Tumor sections of $8-10 \mu \mathrm{m}$ were incubated in xylene and then washed with absolute ethanol. DNA was isolated from the air-dried tissues using the QIAamp ${ }^{\circ}$ DNA FFPE Tissue Kit (QIAGEN, Hilden, Germany) according to the manufacturer's instructions. Screening of mutations was performed by direct sequencing of the PCR products obtained using primer pairs designed to selectively amplify PDGFRA exons 12 and 18 and KIT exons 9, 11, 13 and 17. PCR reactions were performed using $100 \mathrm{ng}$ of DNA with the primers listed in Additional file 1: Table S1. Mutation analysis was assessed by sequencing of PCR products with the same primers used for PCR reactions and the BigDye ${ }^{\bullet}$ Terminator v1.1 cycle sequencing kit (Applied Biosystems). Sample analysis was performed on an ABI PRISM 310 Genetic Analyzer (Applied Biosystems).

\section{Immunohistochemistry}

The expression of DOG1 was investigated by IHC with the anti-DOG1 monoclonal antibody (MoAb; clone K9, Abcam Cambridge, MA). Five $\mu \mathrm{m}$ FFPE sections of each primary tumor were treated according to the staining Dako Autostainer protocol (Burlington, Ontario, Canada). Briefly, sections were incubated with the antiDOG1 MoAb at 1:100 dilution for $30 \mathrm{~min}$ at room temperature. Stained specimens were analyzed by two pathologists and results were scored according to the Allred scoring system, including a semi-quantitative method to reveal the staining intensity $(0=$ negative; $1=$ weak $/$ trace; 2 = moderate; $3=$ strong) and the percentage of positive cells $(0=$ normal cells; $1=\leq 1 \%$; $2=1-10 \% ; 3=11-33 \% ; 4=34-66 \% ; 5=67-100 \%$ ). This grading produced a final score [23] that was reported as negative (score 0 ), weak (score 1-3), moderate (score 4-6) or strong (score 7-8).

\section{Statistical analysis}

Fisher's exact test was used to evaluate differences between independent groups. The $p$-values for differences 
between subgroups were adjusted by a permutational test performed in the multitest SAS STAT procedure. Comparison between independent groups was performed by $t$-test, given the Gaussian distribution of data. Statistical analysis was performed using SAS 9.4 software. Recurrence-free survival (RFS) was defined as the time from the date of operation to the date of recurrence and/or distant metastasis. Patients who survived without recurrence and/or metastasis were censored on the date of the last follow-up. RFS was calculated according to the Kaplan-Meier method and the survival distributions were compared by log-rank test. A $p$-value $<0.05$ was considered statistically significant.

\section{Results}

\section{Demographics}

As shown in Table 1, 59 patients with GISTs were enrolled in the study (57 locally-advanced and 2 metastatic; 31 males (52.5\%) and 28 females (47.5\%), median age $63.3 \pm 14.6$ years). Primary sites included the stomach $(n=39 ; 66.1 \%)$, small $(n=12 ; 20.3 \%)$ and large bowel $(n=4 ; 6.8 \%)$, as well as extra-gastrointestinal sites $(n=4 ; 6.8 \%)$ including the pancreas and retroperitoneum. The histological subtypes included spindlecell $(n=45 ; 76.3 \%)$, epithelial $(n=6 ; 10.2 \%)$ and mixed $(n=8 ; 13.5 \%)$ variants. Mean tumor size was $8.3 \pm 5.5 \mathrm{~cm}$, while the number of mitoses (HPFx50) was $\leq 5$ in 25 (42.4\%), 6-10 in 17 (28.8\%) and $\geq 10$ in 17 (28.8\%) patients. Despite slight variations in CD117 staining intensity, it was considered positive in all patients. Mutational status was available in 53 patients harboring mutations of $\operatorname{KIT}(n=35 ; 66.1 \%)$ and PDGFRA $(n=4 ; 7.5 \%)$ (Fig. 1$)$, whereas in 14 patients $(26.4 \%)$ both genes were WT. The identified hot-spot mutations are listed in Additional file 2: Table S2. The average follow-up was $36+21$ months; $22 \%$ of patients $(n=13)$ had evidence of disease recurrence.

\section{DOG1 expression in GISTs correlates with clinical and pathological features}

Based on the IHC DOG1 expression, 39 patients $(66.1 \%)$ were included in Group A $\left(\mathrm{DOG}^{+}\right)$and 20 $(33.9 \%)$ in $\mathrm{B}\left(\mathrm{DOG1}^{-}\right)$. Representative panels from both groups are included in Fig. 2, showing strong (a), moderate (b) and weak (c) as well as negative (d) cytoplasmic or membranous DOG1 expression. Based on the Allred scoring system, a strong DOG1 expression was demonstrated in 24 Group A patients (Group A1), moderate levels in 12 (Group A2) and a weak expression in 3 patients (Group A3). Levels of DOG1 expression did not correlate with gender, age, primary site, histology, mitoses or mutational status (Table 1). By contrast, tumor size in Group A patients was greater $(10.1 \pm 5.8 \mathrm{~cm})$ than in Group B $(4.7 \pm 1.9 \mathrm{~cm} ; p=0.0002)$, whereas
Table 1 Clinical pathological features of the 59 GIST cases according to DOG1 expression

\begin{tabular}{|c|c|c|c|c|}
\hline \multirow{2}{*}{$\begin{array}{l}\text { Patient } \\
\text { characteristics }\end{array}$} & \multirow{2}{*}{$\begin{array}{l}\text { Cumulative } \\
\text { population }\end{array}$} & \multicolumn{2}{|l|}{$\underline{\mathrm{DOG} 1}$} & \\
\hline & & Positive & Negative & \\
\hline \multicolumn{5}{|l|}{ Sex } \\
\hline Male, n (\%) & $31(52.5 \%)$ & 19 (48.7\%) & $12(60 \%)$ & $p=0.413$ \\
\hline Female, n (\%) & $28(47.5 \%)$ & $20(51.3 \%)$ & $8(40 \%)$ & \\
\hline \multicolumn{5}{|l|}{ Age (years) } \\
\hline Mean $( \pm \mathrm{SD})$ & $63.3( \pm 14.6)$ & $63.1( \pm 16.9)$ & $63.6( \pm 12.8)$ & $p=0.8973$ \\
\hline Median & 67 & 65 & 66.5 & \\
\hline Range & $28-88$ & $28-88$ & $34-82$ & \\
\hline Primary site & & & & $p=0.0652$ \\
\hline Stomach & 39 (66.1\%) & $29(74.3 \%)$ & $10(50 \%)$ & \\
\hline Small intestine & $12(20.3 \%)$ & $8(20.5 \%)$ & $4(20 \%)$ & \\
\hline Large bowel & $4(6.8 \%)$ & $1(2.6 \%)$ & $3(15 \%)$ & \\
\hline Others & $4(6.8 \%)$ & $1(2.6 \%)$ & $3(15 \%)$ & \\
\hline Tumor size $(\mathrm{cm})$ & & & & $p=0.0002$ \\
\hline Mean $( \pm \mathrm{SD})$ & $8.3( \pm 5.5)$ & $10.1( \pm 5.8)$ & $4.7( \pm 1.9)$ & \\
\hline Median & 6 & 8 & 4.7 & \\
\hline Range & $2-20$ & $3-20$ & $2-10$ & \\
\hline Histological subtype & & & & $p=0.2$ \\
\hline Spindle type & $45(76.3 \%)$ & $27(69.2 \%)$ & 18 (90 \%) & \\
\hline Epithelial type & $6(10.2 \%)$ & $5(12.8 \%)$ & $1(5 \%)$ & \\
\hline Mixed type & $8(13.5 \%)$ & $7(18 \%)$ & $1(5 \%)$ & \\
\hline \multicolumn{5}{|l|}{ Mitoses per 50 HPFs } \\
\hline$\leq 5$ & $25(42.4 \%)$ & 15 (38.5 \%) & $10(50 \%)$ & $p=0.54$ \\
\hline $6-10$ & $17(28.8 \%)$ & $13(33.3 \%)$ & $4(20 \%)$ & \\
\hline$\geq 10$ & $17(28.8 \%)$ & $11(28.2 \%)$ & $6(30 \%)$ & \\
\hline \multicolumn{5}{|l|}{ Mutated exon } \\
\hline KIT exon 11 & $30(56.6 \%)$ & $24(68.6 \%)$ & $6(33.5 \%)$ & $p=1$ \\
\hline KIT exon 9 & 2 (3.8 \%) & $2(5.7 \%)$ & 0 & \\
\hline KIT exon 13 & 2 (3.8 \%) & $2(5.7 \%)$ & 0 & \\
\hline KIT exon 17 & 1 (1.9\%) & 0 & 1 (5.5 \%) & \\
\hline PDGFRA exon 12 & $1(1.9 \%)$ & 0 & 1 (5.5 \%) & $p=0.889$ \\
\hline PDGFRA exon 18 & $3(5.6 \%)$ & $2(5.7 \%)$ & $1(5.5 \%)$ & \\
\hline Wild type & $14(26.4 \%)$ & $5(14.3 \%)$ & 9 (50 \%) & $p=0.009$ \\
\hline Not available & 6 & 4 & 2 & \\
\hline
\end{tabular}

HPF high power field of the microscope; $p$-values computed using Fisher exact test or $x^{2}$ test

the frequency of the WT status for both KIT and PDGFRA was lower in Group A than B (14.3\% vs. $50 \% ; p=0.009)$.

\section{DOG1 expression levels and GIST outcome}

DOG1 expression was investigated in relation to a potential predictive role with respect to the onset of recurrence. Nine Group A patients (23\%) and four Group B 


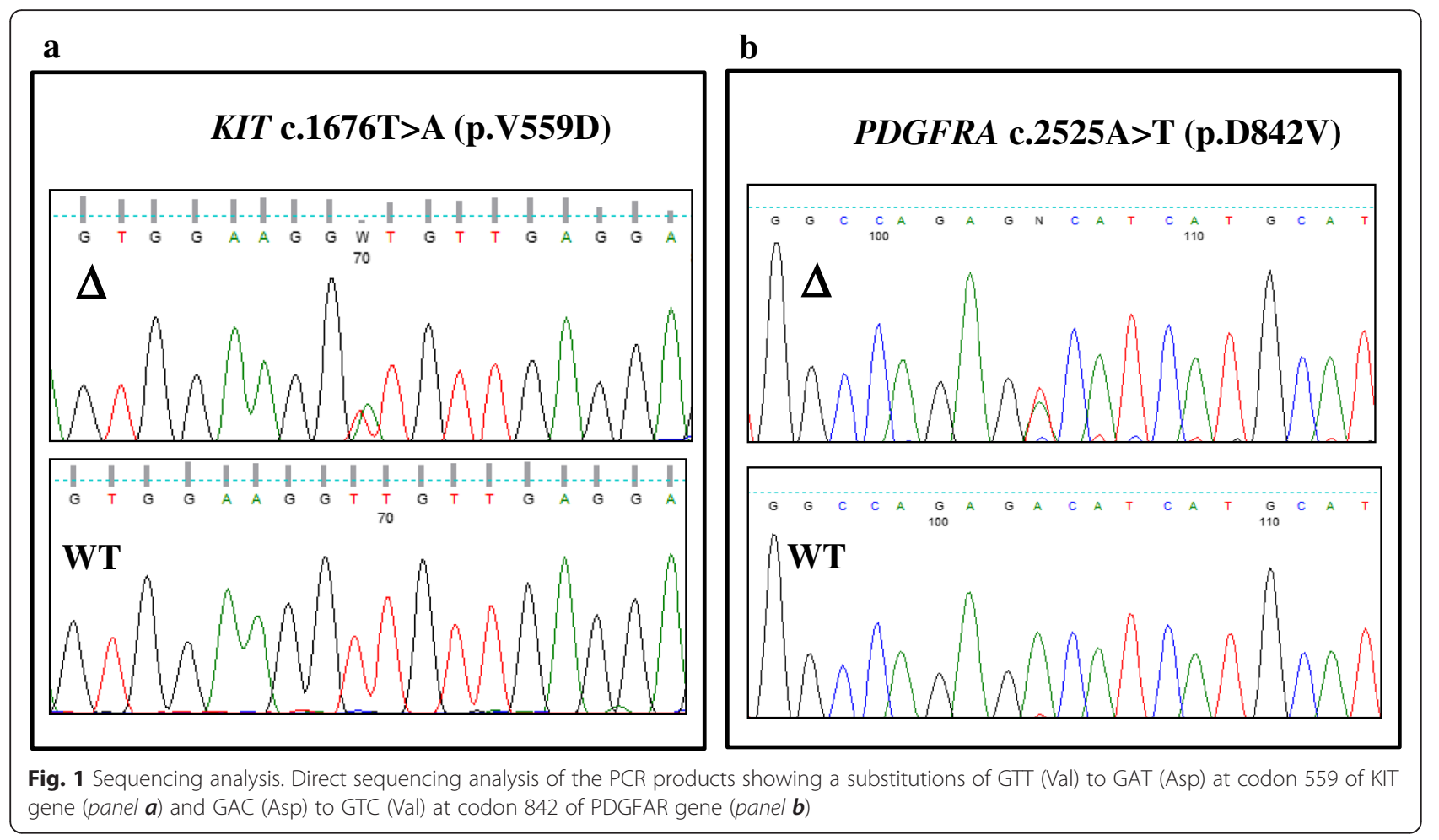

(20 \%) recurred during follow-up, yielding 2-year RFS rates of 84 and $95 \%$ respectively. The cumulative RFS curve in Group A patients was worse, although not significantly so, compared to Group B (Fig. 3a). We also investigated the relationship between DOG1 levels and RFS, and found that Group A1 patients had the worst 2-year (panel b) RFS rate $(80 \% ; 6 / 24)$ as compared to the other groups $(93 \% ; 7 / 35)$. Further analyses were performed to investigate whether the previously described correlation of DOG1 expression with both tumor size and mutational status was associated with the RFS. Therefore, Group A1 patients were subdivided a
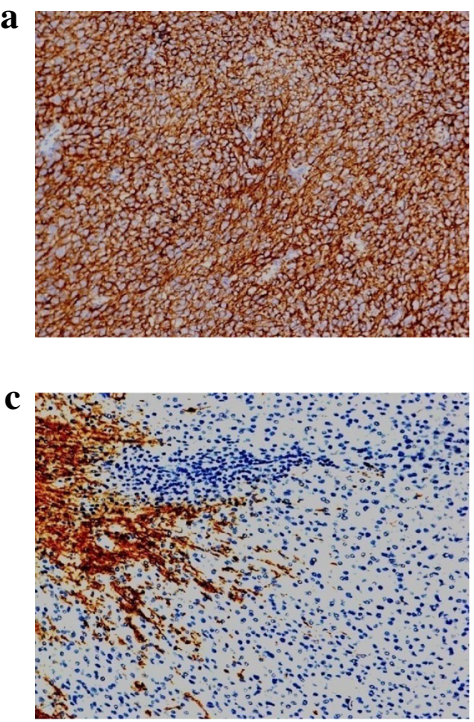

b

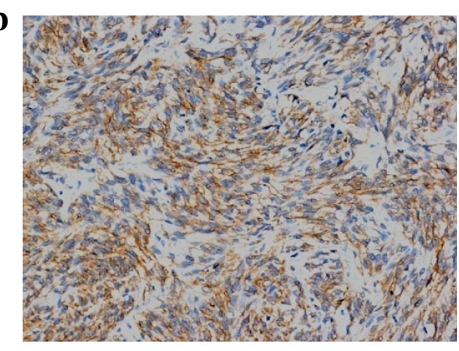

d

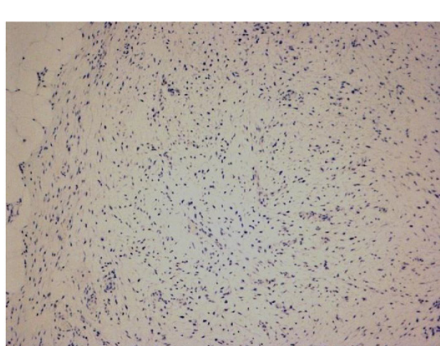

Fig. 2 DOG1 measurement according to the Allred scoring system. Representative panels showing the variable DOG1 expression by $1 \mathrm{HC}$ in patients with GISTs: strong (score: 7-8, panel a), moderate (score: 4-6, b) and weak (score: 1-3, c), while panel $\mathbf{d}$ shows a DOG1 negative specimen. Magnification is 200x in $\mathbf{a}, \mathbf{b}$ and $\mathbf{c}, 100 \mathrm{x}$ in $\mathbf{d}$ 

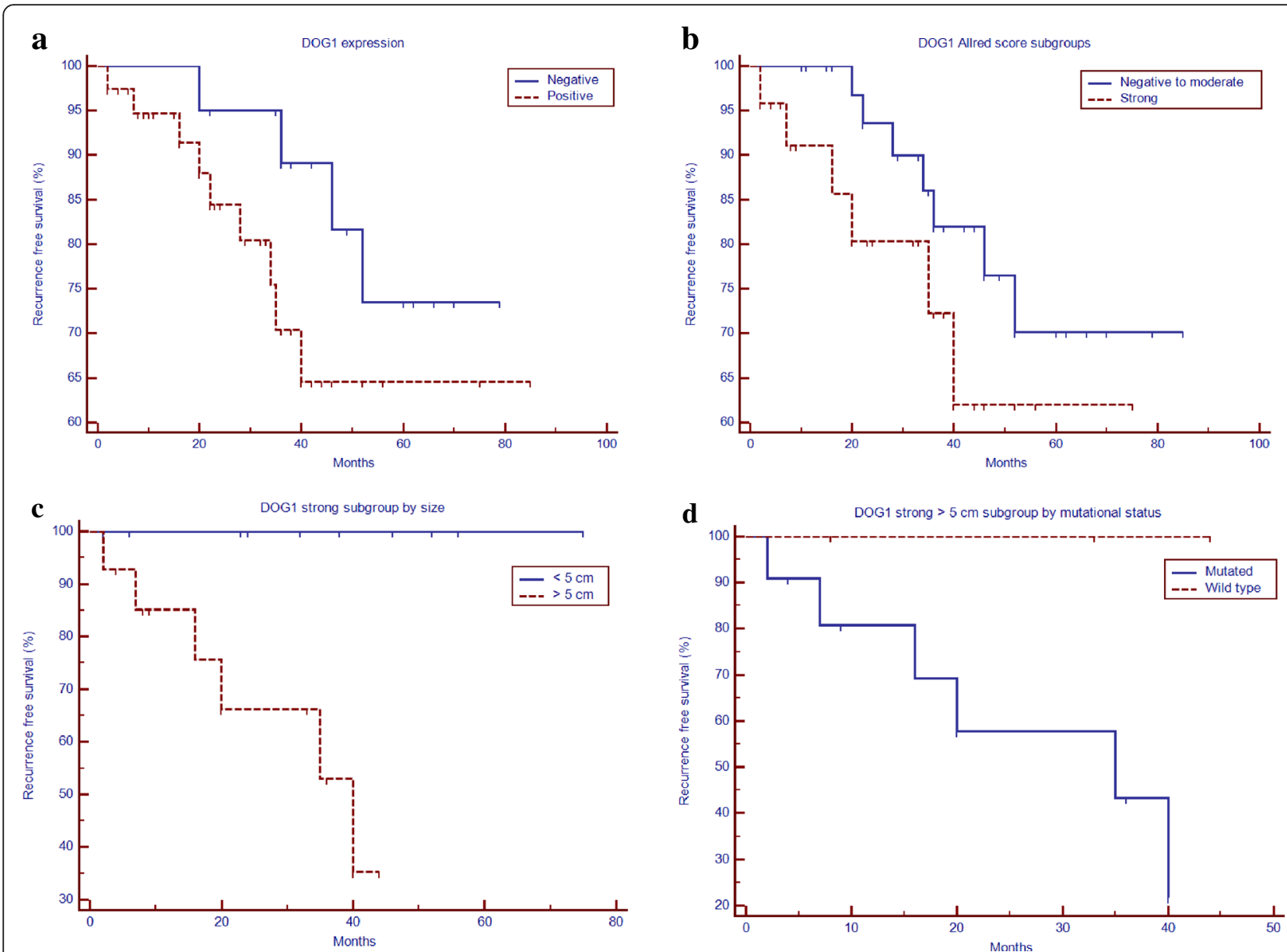

Fig. 3 Kaplan-Meier cumulative RFS. a The 2-year RFS rate of DOG1-positive patients (Group A) was 84 \% (dashed line; $p=0.2$ ) as compared to DOG1-negative patients (95\%; solid line; Group B). Disease recurrence occurred in 9/39 and 4/20 patients, respectively. b Group A patients were divided by the Allred scoring system into 3 sub-groups (A1, A2 and A3) based on the DOG1 expression levels, indicated as strong, moderate and weak, respectively. The 2-year RFS rate for A1 patients was $80 \%$ (dashed line) compared to $93 \%$ for A2 $+\mathrm{A} 3+\mathrm{B}$ patients (solid line; $p=0.2$ ). Disease recurrence occurred in 6/24 and 7/35 patients, respectively. c Group A1 patients were divided by tumor size greater or smaller than $5 \mathrm{~cm}$. The 2-year RFS rate for patients bearing tumors $>5 \mathrm{~cm}$ was $66 \%$ (dashed line) with $6 / 14$ events compared to $100 \%$ for those with tumors $>5 \mathrm{~cm}$ (ten patients) (solid line; $p=0.01$ ). $\mathbf{d}$ The 14 Group A1 patients with tumor size $>5 \mathrm{~cm}$ were subdivided by mutational status, and the 2-year RFS rate for those $(n=11)$ harboring mutations was $58 \%$ (solid line) compared to $100 \%$ for the 3 WT patients (dashed line, $p=0.16$ ). Recurrence occurred in 6/11 Group A1 patients

by tumor size greater $(n=14)$ or smaller $(n=10)$ than $5 \mathrm{~cm}$. As shown in panel c, the Kaplan-Meier survival curve revealed 2-year RFS rates of $66 \%$ (6/14 events) and $100 \%$ respectively $(p=0.01)$. Moreover, among A1 patients with a tumor size $>5 \mathrm{~cm}$ (panel d), those carrying a KIT or PDGFRA mutation $(n=11)$ had a worse prognosis than the WT $(n=3)$, the 2 -year RFS rates being 58 and $100 \%$ respectively. The trend to statistical significance ( $p=0.16)$ was, however, influenced by the sample size.

\section{Discussion}

GISTs are rare tumors with morphological, histological and molecular features that strongly influence both the outcome and risk of recurrence. Since the discovery of the role of oncogenic mutations of KIT and PDGFRA, targeted therapy with TKI has significantly increased the OS in the majority of patients. However, WT GISTs or those harboring rare mutations often experience progression or recurrence and so a better risk stratification is needed in order to plan adequate therapeutic strategies.

Measurement of DOG1 expression by IHC has been associated with a higher diagnostic sensitivity and specificity than CD117, allowing the diagnosis of GISTs in about $30 \%$ of CD117-negative patients [18]. Its expression has been described in both normal and malignant tissues, although its prognostic role is still being debated. The DOG1 protein mediates the receptor-activated chloride current whose levels modulate the cell proliferation by affecting the retinoblastoma $(\mathrm{Rb})$ tumor 
suppressor protein phosphorylation [14, 24, 25], or by activating the MEK/ERK pathway [15]. In addition, xenograft DOG1 $^{-1-}$ models of GISTs show an impaired cell proliferation as a consequence of the decreased IGF binding protein-5 levels [16], that inhibit IGF-mediated downstream signals by trapping both IGF1 and IGF2 [26]. These findings suggest that DOG1 over-expression provides a proliferative advantage to malignant stromal cells, and increased levels could negatively influence prognosis.

Here, we describe results from an observational study based on evaluation of the clinical, pathological and molecular features of 59 GIST patients and any correlations with DOG1 expression. Approximately $66 \%$ of CD117 ${ }^{+}$ samples showed a strong DOG1 expression, in agreement with previous studies describing its variable accumulation in 60-99 \% malignant cells. The reported variability in DOG1 expression was mostly attributed to different monoclonal antibodies used for IHC analyses, as well as to the intrinsic characteristics of the specimens [10, 21, 27]. In accordance with previous studies [19-21, 28], our data showed that DOG1 expression is unrelated to gender, age, primary site, histological subtypes and mitoses, although a significant correlation was demonstrated with large tumors harboring an unfavorable mutational status. Tumor size is already considered a prognostic factor for the definition of high-risk disease [29-31]. However, the prognostic role of the mutational status is still under debate and not included in the current risk stratification systems. It is noteworthy that the presence of the homozygous KIT exon-11 mutation predicts an aggressive disease course, in particular when deletions affect both codons 557-558 [32]. By contrast, the majority of PDGFRA mutated GISTs show a benign course [33]. Our data support those recently published in a meta-analysis on 1487 patients [34], proving that GISTs bearing KIT mutations have a significantly poorer prognosis than either PDGFRA mutated or WT GISTs. Moreover, Rìs-Moreno et al. reported that the WT genotype was prevalent in DOG1 ${ }^{-} / \mathrm{CD} 117^{-}$patients [35]. We demonstrated a more favorable post-operative 2-year RFS rate in DOG1-negative patients than DOG1-positive patients $(p=0.02)$. These findings were in line with previous results [36] that reported a significant association between DOG1 expression and high-risk tumors. We stratified DOG1 positive patients in relation to the Allred scoring system to identify those with a higher risk of recurrence; in our study patients with a strong DOG1 expression, tumor size $\geq 5 \mathrm{~cm}$ and mutations of $K I T$ or PDGFRA had a worse prognosis.

The genetic landscape of GIST patients should be further investigated. In particular, given the correlation between DOG1 expression and the activation of the downstream RAS/RAF/MEK/ERK signaling pathway, the clinical significance of activating $R A S$ mutations remains to be better elucidated for its therapeutic relevance, as already widely investigated in other tumors [37].

\section{Conclusions}

In conclusion, in our patients a high DOG1 expression correlated with an aggressive malignant phenotype of GISTs. Thus, measurement of DOG1 expression would be helpful in clinical practice to predict the recurrence risk in GIST patients. We believe that the Allred scoring system could be integrated in current risk stratification systems to achieve a better identification of patients at increased risk of recurrence.

\section{Availability of data and materials}

The datasets supporting the conclusions of this article are included within the article and its additional files.

\section{Additional files}

\begin{abstract}
Additional file 1: Table S1. Polymerase Chain Reaction primers, product size and reaction conditions for amplification and direct sequencing for assay of KIT and PDGFRA genes. (DOC 38 kb)
\end{abstract}

Additional file 2: Table S2. Genotype of GIST patients carrying mutations. (DOCX $19 \mathrm{~kb}$ )

\begin{abstract}
Abbreviations
ANO1: anoctamin-1; DOG1: discovered on GIST-1; FFPE: formalin-fixed, paraffin-embedded; GISTs: gastrointestinal stromal tumor; HPFs: high-power fields; IGF: insulin-like growth factor; IHC: immunohistochemistry; KIT: v-kit Hardy-Zuckerman 4 feline sarcoma viral oncogene homolog; OS: overall survival; PCR: polymerase chain reaction; PDGFRA: platelet-derived growth factor receptor, alpha polypeptide; Rb: retinoblastoma; RFS: recurrence-free survival; SDH: succinate dehydrogenase; TKI: tyrosine kinase inhibitors; TMEM16A: transmembrane member 16A; WT: wild-type.
\end{abstract}

Competing interests

The authors declare that they have no competing interests.

Authors' contributions

FMR acquired data, carried out data analysis, participated in figure and table preparation and drafted the manuscript. RP and AM performed the histological review of all tissue samples and IHC assay and participated in figure preparation. NR carried out the molecular genetic studies and participated in drafting the manuscript. MC contributed to data analysis, interpretation of data and figure preparation. MT designed the study, performed the statistical analysis and interpretation of data, helped to draft the manuscript and participated in figure and table preparation. FS conceived of the study, drafted the manuscript and revised it critically for important intellectual content. All authors read and approved the final manuscript.

\section{Authors' information}

FMR (M.D.) is attending the Postgraduate Specialization School in Oncology at the University of Bari "Aldo Moro". RP (M.D., Ph.D.) coordinates the genetic group at the Laboratory of Molecular Medicine at the Department of Biomedical Sciences and Clinical Oncology, University of Bari "Aldo Moro". AM (M.D., Ph.D.) is Assistant Professor at the Department of Pathological Anatomy at the University of Bari "Aldo Moro". NR (Ph.D.) is Associate Professor of Medical Genetics, Department of Biomedical Sciences and Human Oncology, University of Bari "A. Moro". MC (M.D.) is fellow at the Laboratory of Molecular Medicine at the Department of Biomedical Sciences and Clinical Oncology, University of Bari "Aldo Moro". MT (M.D., Ph.D.) is Assistant Professor at the Medical Oncology Unit, Department of Biomedical 
Sciences and Clinical Oncology, University of Bari "Aldo Moro". FS (M.D.) is Full Professor of Internal Medicine and Clinical Oncology and Chief of the Hospital Clinical Division of Medical Oncology, President of the Postgraduate Specialization School in Oncology and Head of the Laboratory of Molecular Medicine at the Department of Biomedical Sciences and Clinical Oncology, University of Bari "Aldo Moro".

\section{Acknowledgments}

This work was supported by a grant from the Italian Association for Cancer Research (AIRC, IG11647). The Authors are indebted to Mary Victoria Pragnell for editorial assistance.

\section{Author details}

'Department of Biomedical Sciences and Human Oncology, University of Bari "A. Moro", Piazza Giulio Cesare, 11-70124 Bari, Italy. ${ }^{2}$ University San Raffaele Rome, Interinstitutional Multidisciplinary BioBank (BioBIM), IRCCS San Raffaele Pisana, Rome, Italy. ${ }^{3}$ Department of Pathology, University of Bari "A. Moro", Bari, Italy. ${ }^{4}$ Division of Medical Genetics, Department of Biomedical Sciences and Human Oncology, University of Bari "A. Moro", Bari, Italy.

\section{Received: 5 September 2015 Accepted: 3 February 2016}

\section{Published online: 11 February 2016}

\section{References}

1. Hirota S, Isozaki K, Moriyama Y, Hashimoto K, Nishida T, Ishiguro S, et al. Gain-of-function mutations of c-kit in human gastrointestinal stromal tumors. Science. 1998;279(5350):577-80.

2. Heinrich MC, Corless CL, Duensing A, McGreevey L, Chen CJ, Joseph N, et al. PDGFRA activating mutations in gastrointestinal stromal tumors. Science. 2003:299(5607):708-10.

3. Lasota J, Miettinen M. Clinical significance of oncogenic KIT and PDGFRA mutations in gastrointestinal stromal tumours. Histopathology. 2008;53(3):245-66

4. Jones RL. Practical aspects of risk assessment in gastrointestinal stromal tumors. J Gastrointest Cancer. 2014;45(3):262-7.

5. Fletcher CD, Berman JJ, Corless C, Gorstein F, Lasota J, Longley BJ, et al. Diagnosis of gastrointestinal stromal tumors: a consensus approach. Hum Pathol. 2002;33(5):459-65.

6. Nannini M, Astolfi A, Urbini M, Indio V, Santini D, Heinrich $M C$, et al. Integrated genomic study of quadruple-WT GIST (KIT/PDGFRA/SDH/RAS pathway wild-type GIST). BMC Cancer. 2014;14:685.

7. Miranda C, Nucifora M, Molinari F, Conca E, Anania MC, Bordoni A, et al. KRAS and BRAF mutations predict primary resistance to imatinib in gastrointestinal stromal tumors. Clin Cancer Res. 2012;18(6):1769-76.

8. Origone P, Gargiulo S, Mastracci L, Ballestrero A, Battistuzzi L, Casella C, et al, Molecular characterization of an Italian series of sporadic GISTs. Gastric Cancer. 2013;16(4):596-601.

9. Lasota J, Xi L, Coates T, Dennis R, Evbuomwan MO, Wang ZF, et al. No KRAS mutations found in gastrointestinal stromal tumors (GISTs): molecular genetic study of 514 cases. Mod Pathol. 2013;26(11):1488-91.

10. Hechtman JF, Zehir A, Mitchell T, Borsu L, Singer S, Tap W, et al. Novel oncogene and tumor suppressor mutations in KIT and PDGFRA wild type gastrointestinal stromal tumors revealed by next generation sequencing. Genes Chromosomes Cancer. 2015:54(3):177-84.

11. Cole WC. ANO1-ther brick in the wall-role of Ca2 +-activated $\mathrm{Cl}$ - channels of interstitial cells of Cajal in cholinergic motor control of gastrointestinal smooth muscle. J Physiol. 2011;589(Pt 19):4641-2.

12. Katoh M. FLJ10261 gene, located within the CCND1-EMS1 locus on human chromosome 11q13, encodes the eight-transmembrane protein homologous to C12orf3, C11 orf25 and FLJ34272 gene products. Int J Oncol. 2003;22(6):1375-81.

13. Novelli M, Rossi S, Rodriguez-Justo M, Taniere P, Seddon B, Toffolatti L, et al. DOG1 and CD117 are the antibodies of choice in the diagnosis of gastrointestinal stromal tumours. Histopathology. 2010;57(2):259-70.

14. Stanich JE, Gibbons SJ, Eisenman ST, Bardsley MR, Rock JR, Harfe BD, et al. Ano1 as a regulator of proliferation. Am J Physiol Gastrointest Liver Physiol. 2011:301(6):G1044-51.

15. Duvvuri U, Shiwarski DJ, Xiao D, Bertrand C, Huang X, Edinger RS, et al. TMEM16A induces MAPK and contributes directly to tumorigenesis and cancer progression. Cancer Res. 2012;72(13):3270-81.
16. Simon S, Grabellus F, Ferrera L, Galietta L, Schwindenhammer B, Mühlenberg $\mathrm{T}$, et al. DOG1 regulates growth and IGFBP5 in gastrointestinal stromal tumors. Cancer Res. 2013;73(12):3661-70.

17. Wada T, Tanabe S, Ishido K, Higuchi K, Sasaki T, Katada C, et al. DOG1 is useful for diagnosis of KIT-negative gastrointestinal stromal tumor of stomach. World J Gastroenterol. 2013;19(47):9133-6.

18. Liegl B, Hornick JL, Corless CL, Fletcher CD. Monoclonal antibody DOG1.1 shows higher sensitivity than KIT in the diagnosis of gastrointestinal stromal tumors, including unusual subtypes. Am J Surg Pathol. 2009;33(3):437-46.

19. Miettinen M, Wang ZF, Lasota J. DOG1 antibody in the differential diagnosis of gastrointestinal stromal tumors: a study of 1840 cases. Am J Surg Pathol. 2009;33(9):1401-8

20. Sui $X L$, Wang $H$, Sun XW. Expression of DOG1, CD117 and PDGFRA in gastrointestinal stromal tumors and correlations with clinicopathology. Asian Pac J Cancer Prev. 2012;13(4):1389-93.

21. Espinosa I, Lee CH, Kim MK, Rouse BT, Subramanian S, Montgomery K, et al. A novel monoclonal antibody against DOG1 is a sensitive and specific marker for gastrointestinal stromal tumors. Am J Surg Pathol. 2008;32(2):210-8.

22. Sözütek D, Yanık S, Akkoca AN, Sözütek A, Ozdemir ZT, Avşar CU, et al. Diagnostic and prognostic roles of DOG1 and Ki-67, in GIST patients with localized or advanced/metastatic disease. Int J Clin Exp Med. 2014:7(7):1914-22

23. Allred DC, Clark GM, Elledge R, Fuqua SA, Brown RW, Chamness GC, et al. Association of p53 protein expression with tumor cell proliferation rate and clinical outcome in node-negative breast cancer. J Natl Cancer Inst. 1993:85(3):200-6.

24. Yang YD, Cho H, Koo JY, Tak MH, Cho Y, Shim WS, et al. TMEM16A confers receptor-activated calcium-dependent chloride conductance. Nature. 2008:455(7217):1210-5.

25. Hartzell C, Putzier I, Arreola J. Calcium-activated chloride channels. Annu Rev Plant Physiol Plant Mol Biol. 2005;67:719-58.

26. Beattie J, Allan GJ, Lochrie JD, Flint DJ. Insulin-like growth factorbinding protein-5 (IGFBP-5): a critical member of the IGF axis. Biochem J. 2006;395(1):1-19.

27. Hwang DG, Qian X, Hornick JL. DOG1 antibody is a highly sensitive and specific marker for gastrointestinal stromal tumors in cytology cell blocks. Am J Clin Pathol. 2011;135(3):448-53.

28. Kang YN, Jung HR, Hwang I. Clinicopathological and immunohistochemical features of gastointestinal stromal tumors. Cancer Res Treat. 2010:42(3):135-43.

29. Franquemont DW. Differentiation and risk assessment of gastrointestinal stromal tumors. Am J Clin Pathol. 1995;103(1):41-7.

30. Agaimy A, Märkl B, Arnholdt H, Hartmann A, Schneider-Stock R, Chetty R. Sporadic segmental interstitial cell of Cajal hyperplasia (microscopic GIST) with unusual diffuse longitudinal growth replacing the muscularis propria: differential diagnosis to hereditary GIST syndromes. Int J Clin Exp Pathol. 2010:3(5):549-56.

31. Maki RG. The rule of fives, a simple way to stratify risk for primary gastrointestinal stromal tumors (GIST). Clin Sarcoma Res. 2012;2(1):21.

32. Miettinen M, Lasota J. KIT (CD117): a review on expression in normal and neoplastic tissues, and mutations and their clinicopathologic correlation. Appl Immunohistochem Mol Morphol. 2005;13(3):205-20.

33. Lasota J, Dansonka-Mieszkowska A, Sobin LH, Miettinen M. A great majority of GISTs with PDGFRA mutations represent gastric tumors of low or nomalignant potential. Lab Invest. 2004;84(7):874-83.

34. Zong L, Chen P. Prognostic value of KIT/PDGFRA mutations in gastrointestinal stromal tumors: a meta-analysis. World J Surg Oncol. 2014;12:71

35. Ríos-Moreno MJ, Jaramillo S, Pereira Gallardo S, Vallejo A, Mora M, García-Escudero A, et al. Gastrointestinal stromal tumors (GISTs): CD117, DOG-1 and PKC $\theta$ expression. Is there any advantage in using several markers? Pathol Res Pract. 2012;208(2):74-81.

36. Abdel-Hadi M, Bessa SS, Hamam SM. Evaluation of the novel monoclonal antibody against DOG1 as a diagnostic marker for gastrointestinal stromal tumors. J Egypt Natl Canc Inst. 2009;21(3):237-47.

37. Pao W, Wang TY, Riely GJ, Miller VA, Pan Q, Ladanyi M, et al. KRAS mutations and primary resistance of lung adenocarcinomas to gefitinib or erlotinib. PLoS Med. 2005:2(1):e17. 